fractures. Considering the nearly equal risk of secondary surgical procedures and the modest benefit in functional outcome, should we abandon the use of total hip arthroplasty in the treatment of hip fractures? Even if the benefits seem smaller than we previously thought, patients with high physical demands and a long remaining life expectancy should probably still be considered for treatment with total hip arthroplasty. Yet the expected remaining lifetime of those patients who potentially could benefit most from a total hip arthroplasty is much longer than the 2-year follow-up period used in the HEALTH trial. However, the number of secondary procedures after hemiarthroplasty may increase with longer follow-up. Therefore, one hopes that the HEALTH investigators will be able to provide long-term results from their trial in the future. Such data would be an even more important contribution to the knowledge base that supports hip-fracture treatment. There is still a need for large randomized, controlled trials or registry-based randomized clinical trials with greater numbers of patients in order to identify how factors such as patient activity level, biologic age, and remaining life expectancy influence the risk of secondary surgical procedures and functional outcome after hemiarthroplasty and total hip arthroplasty. Until then, in light of the results of the trial by Bhandari et al., we should probably be restrictive in the selection criteria for total hip arthroplasty for patients with hip fractures.
Disclosure forms provided by the author are available with the full text of this editorial at NEJM.org.

From the Department of Orthopedic Surgery, Haukeland University Hospital, and the Institute of Clinical Medicine, University of Bergen — both in Bergen, Norway.

1. Parker M, Johansen A. Hip fracture. BMJ 2006;333:27-30.

2. Hip fracture: management. Clinical guideline CG124. London: National Institute for Health and Clinical Excellence, June 2011 (https://www.nice.org.uk/Guidance/CG124).

3. Management of hip fractures in the elderly: summary. Rosemont, IL: American Academy of Orthopedic Surgeons, 2019 (https://www.aaos.org/cc_files/aaosorg/research/guidelines/ hipfxsummaryofrecommendations.pdf).

4. Hopley C, Stengel D, Ekkernkamp A, Wich M. Primary total hip arthroplasty versus hemiarthroplasty for displaced intracapsular hip fractures in older patients: systematic review. BMJ 2010;340:c2332.

5. Burgers PTPW, Van Geene AR, Van den Bekerom MPJ, et al. Total hip arthroplasty versus hemiarthroplasty for displaced femoral neck fractures in the healthy elderly: a meta-analysis and systematic review of randomized trials. Int Orthop 2012;36:1549-60. 6. Lewis DP, Wæver D, Thorninger R, Donnelly WJ. Hemiarthroplasty vs total hip arthroplasty for the management of displaced neck of femur fractures: a systematic review and metaanalysis. J Arthroplasty 2019;34(8):1837-1843.e2.

7. The HEALTH Investigators. Total hip arthroplasty or hemiarthroplasty for hip fracture. N Engl J Med 2019;381:2199-208.

8. Tsikandylakis G, Mohaddes M, Cnudde P, Eskelinen A, Kärrholm J, Rolfson O. Head size in primary total hip arthroplasty. EFORT Open Rev 2018;3:225-31.

9. Jobory A, Kärrholm J, Overgaard S, et al. Reduced revision risk for dual-mobility cup in total hip replacement due to hip fracture: a matched-pair analysis of 9,040 cases from the Nordic Arthroplasty Register Association (NARA). J Bone Joint Surg Am 2019;101:1278-85.

10. Hip, knee \& shoulder arthroplasty: 2019 annual report. Adelaide, SA: Australian Orthopaedic Association National Joint Replacement Registry, 2019.

DOI: 10.1056/NEJMe1913800

Copyright (c) 2019 Massachusetts Medical Society.

\title{
Parting the Clouds over Typhoid with a New Conjugate Vaccine
}

\author{
Florian Marks, Ph.D., and Jerome H. Kim, M.D.
}

Typhoid fever is caused by fecal-oral transmission of Salmonella enterica serovar Typhi (S. Typhi). It has been a deadly companion to mankind for centuries, affecting 10.9 million persons and resulting in an estimated 116,800 deaths per year. ${ }^{1}$ Although vaccines against typhoid have been available for more than a century and have been shown to be protective, ${ }^{2,3}$ the approved vaccines (injectable Vi polysaccharide and oral, live-attenuated Ty21a typhoid vaccines) have not been useful in populations with a high typhoid burden, particularly in young children. ${ }^{4}$ To address this shortfall, the Bill and Melinda Gates Foundation, as well as other donors, has supported the development of new typhoid conjugate vaccines (TCVs), generated data on disease burden, and coordinated with international stakeholders to introduce the vaccine in countries where typhoid fever is endemic.

Typbar-TCV was developed by Bharat Biotech International in India and was prequalified by the World Health Organization (WHO) ${ }^{3}$ on the basis of immunogenicity and evidence of protection (55\% efficacy) in a typhoid human challenge model. ${ }^{5}$ In this issue of the Journal, Shakya et al. ${ }^{6}$ report that this vaccine was immunogenic and efficacious against blood culture-confirmed ty- 
phoid fever, with an estimated vaccine efficacy of $81.6 \%$ at 12 months, in a trial involving children who were between 9 months and 16 years of age.

Data from African and Asian surveillance studies were used by the WHO Strategic Advisory Group of Experts to recommend the use of TCVs in countries where typhoid fever is endemic. ${ }^{2,4}$ Subsequently, TCVs were added to vaccines subsidized by Gavi, the Vaccine Alliance. The Typbar-TCV vaccine is already commercially available in India. Other large studies, such as the TCV introduction program in Navi Mumbai, India (ClinicalTrials.gov number, NCT03554213), are currently ongoing, ${ }^{7}$ and introduction of the vaccine through Gavi subsidies in Asian and African countries is also under way. Ongoing and planned clinical trials are geared toward systematic assessment of vaccine performance and addressing unanswered scientific questions regarding effectiveness, herd immunity, costeffectiveness, and the effect of the vaccine on antimicrobial resistance. The trial by Shakya and colleagues, combined with evidence of safety and immunogenicity in other trials, ${ }^{7}$ provides support for broader introduction of TCVs in countries where typhoid is endemic. The national and international stakeholders involved in decisions regarding the introduction of TCV await results from longer-term follow-up to determine whether protection elicited by TCVs exceeds that of existing Vi polysaccharide and live-attenuated oral vaccines.

Typhoid fever has been successfully treated with antimicrobial agents since early in the antimicrobial era, but sustained antibiotic pressure through large-scale (over)use has created multidrug-resistant and extensively drug-resistant (XDR) typhoid strains that have spread in India, Bangladesh, and Pakistan. ${ }^{8}$ Patients with severe $S$. Typhi infection caused by a resistant strain often have prolonged hospital stays and limited treatment options. Moreover, the presenting signs and symptoms of typhoid fever are nonspecific, and diagnostic testing is hampered by the poor sensitivity and limited availability of existing tests, including blood cultures (which, although insensitive, are considered to be the best available test for typhoid fever), particularly in resourcelimited areas. Thus, patients with suspected typhoid fever in areas where typhoid is endemic frequently receive antimicrobial agents from health care providers, and unnecessary antimicrobial pressure is added to resident bacterial populations. To date, XDR typhoid is confined to India, Bangladesh, and Pakistan, where newer antibiotics are available. ${ }^{8}$ Yet, it is easy to envision that the introduction of XDR S. Typhi strains into Africa, with underresourced health care systems and a lack of treatment options, could lead to a scenario involving considerable morbidity and mortality.

Additional large-scale trials are under way to provide data on vaccine performance in other geographic areas. The Typhoid Vaccine Acceleration Consortium (TyVAC) is evaluating TCV in Dhaka, Bangladesh (in a cluster-randomized trial), Blantyre, Malawi (in an individually randomized trial), and Ouagadougou, Burkina Faso (in coadministration studies). ${ }^{9}$ Researchers in the THECA (Effect of a Novel Typhoid Conjugate Vaccine in Africa: A Multicenter Study in Ghana and the Democratic Republic of the Congo) trial are in close alignment with the TyVAC, conducting a cluster-randomized trial in Agogo, Ghana, and a large-scale trial of vaccine effectiveness in Kisantu, Democratic Republic of Congo. ${ }^{10}$ This compendium of data for stakeholders in the introduction of vaccine may be used to target interventions to protect as many persons as possible given the existing constraints on resources.

Shakya and colleagues have made an important contribution to the global fight against $S$. Typhi infection, but global health is about impact. If further studies support and extend these results, how can a corresponding reduction in the burden of typhoid disease and death be achieved most efficiently?

Disclosure forms provided by the authors are available with the full text of this editorial at NEJM.org.

From the International Vaccine Institute, Seoul, South Korea (F.M., J.H.K.); and the University of Cambridge, Cambridge, United Kingdom (F.M.).

1. GBD 2017 Typhoid and Paratyphoid Collaborators. The global burden of typhoid and paratyphoid fevers: a systematic analysis for the Global Burden of Disease Study 2017. Lancet Infect Dis 2019;19:369-81

2. World Health Organization. Meeting of the strategic Advisory Group of Experts on immunization, October 2017 — conclusions and recommendations. Wkly Epidemiol Rec 2017;92: 729-47

3. World Health Organization. Typhoid vaccine prequalified. January 3, 2019 (http://www.who.int/medicines/news/2017/ WHOprequalifies-breakthrough-typhoid-vaccine/en/).

4. World Health Organization. Typhoid vaccines: WHO position paper, March 2018 - recommendations. Vaccine 2019;37: 214-6. 
5. Jin C, Gibani MM, Moore M, et al. Efficacy and immunogenicity of a Vi-tetanus toxoid conjugate vaccine in the prevention of typhoid fever using a controlled human infection model of Salmonella Typhi: a randomised controlled, phase $2 \mathrm{~b}$ trial. Lancet 2017;390:2472-80.

6. Shakya M, Colin-Jones R, Theiss-Nyland $\mathrm{K}$, et al. Phase 3 efficacy analysis of a typhoid conjugate vaccine trial in Nepal. N Engl J Med 2019;381:2209-18.

7. World Health Organization. Global Advisory Committee on Vaccine Safety, 5-6 December 2018. Wkly Epidemiol Rec 2019;4: 45-52.

8. Levine MM, Simon R. The gathering storm: is untreatable typhoid fever on the way? MBio 2018;9(2):e00482-e18.

9. Meiring JE, Gibani M. The Typhoid Vaccine Acceleration
Consortium (TyVAC): vaccine effectiveness study designs: accelerating the introduction of typhoid conjugate vaccines and reducing the global burden of enteric fever - report from a meeting held on 26-27 October 2016, Oxford, UK. Vaccine 2017;35: 5081-8

10. European \& Developing Countries Clinical Trials Partnership (EDCTP). Effect of a novel typhoid conjugate vaccine in Africa: a multicentre study in Ghana and the Democratic Republic of the Congo (http://www.edctp.org/projects-2/edctp2 -projects/strategic-actions-supporting-large-scale-clinical -trials-2017/).

DOI: 10.1056/NEJMe1914695

Copyright (c) 2019 Massachusetts Medical Society. 